\title{
Quand Emmanuel Macron veut réparer le lien entre l'Eglise et l'Etat. La question de l'autorité dans les commentaires YouTube
}

When Emmanuel Macron wants to repair the link between Church and State. The question of authority in YouTube comments

\section{Laetitia Gern}

\section{(2) OpenEdition} Journals

\section{Electronic version}

URL: http://journals.openedition.org/aad/4976

DOI: $10.4000 /$ aad. 4976

ISSN: 1565-8961

\section{Publisher}

Université de Tel-Aviv

\section{Electronic reference}

Laetitia Gern, "Quand Emmanuel Macron veut réparer le lien entre l'Eglise et l'Etat. La question de l'autorité dans les commentaires YouTube", Argumentation et Analyse du Discours [Online], 26 | 2021 Online since 13 April 2021, connection on 16 April 2021. URL: http://journals.openedition.org/aad/ 4976 ; DOI: https://doi.org/10.4000/aad.4976

This text was automatically generated on 16 April 2021.

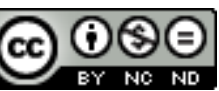

Argumentation \& analyse du discours est mis à disposition selon les termes de la licence Creative Commons Attribution - Pas d'Utilisation Commerciale - Pas de Modification 4.0 International. 


\title{
Quand Emmanuel Macron veut réparer le lien entre l'Eglise et l'Etat. La question de l'autorité dans les commentaires YouTube
}

\author{
When Emmanuel Macron wants to repair the link between Church and State. \\ The question of authority in YouTube comments
}

Laetitia Gern

1 En fondant notre propos sur les travaux pionniers de Susan Herring $(2011,2016)$, nous proposons de nous pencher sur la notion d'autorité sur YouTube telle qu'elle ressort de l'espace des commentaires réservé aux internautes. On sait que la plateforme digitale YouTube est désormais un lieu de partage, d'expression et de mise en visibilité d'arguments politiques (Burgess et Green 2009, Burger 2019, Burger, Thornborrow et Fitzgerald 2017). En partant d'une intervention controversée du Président de la République, le 9 avril 2018 lors de la Conférence des évêques de France au collège des Bernardins, on examinera par quels procédés discursifs et argumentatifs les utilisateurs de la plateforme s'attaquent à l'autorité présidentielle, ou au contraire la défendent contre les attaques. En même temps, dans ce rapport aux dires du Président, on verra par quelles stratégies de positionnement et par quelles procédures argumentatives ils construisent leur propre autorité de commentateurs politiques.

Dans son discours, Emmanuel Macron regrette que le lien entre Église et État se soit abîmé et exprime son désir de le restaurer. Symbole de la laïcité (Weil 2007 : 9), la loi de Séparation, promulguée le 9 décembre 1905 est « l'aboutissement d'un long processus de laïcisation et de sécularisation, engagé depuis la Révolution française $»^{1}$. Cent quinze après, la déclaration du Président Emmanuel Macron sur la réparation du lien entre l'Église et l'État reste étroitement liée à la question du rapport à la loi de $1905^{2}$ et provoque de nombreuses réactions dans l'espace public. Plusieurs personnalités politiques l'accusent de porter atteinte au principe laïque et déversent une " pluie de critiques » sur les réseaux sociaux ${ }^{3}$ (Ardid, Bret et Hubert 2020 : 122-123). Nous nous 
concentrerons sur les réactions d'internautes à un extrait du discours présidentiel publié par la chaîne YouTube "Le Monde » le 10 avril 2018 intitulé «Pour Emmanuel Macron, "le lien entre l'Église et l'État s'est abîmé" "44. Les 540 commentaires publiés en réaction à cette vidéo nous donnent accès à une parole citoyenne qui thématise son rapport à la légitimité et à la crédibilité du Président de la République et construit une identité qui lui est propre.

\section{Autorité, légitimité et crédibilité}

\subsection{Réflexions préliminaires}

Les travaux de Patrick Charaudeau éclairent le lien entre l'autorité et le processus de soumission de l'autre. Selon lui, "l'autorité est une position dans un processus d'influence qui donne au sujet le droit de soumettre l'autre avec l'acceptation de celuici » (2005a : 52-53). L'enjeu de l'autorité, qui vient se "surajouter à la légitimité » comprise comme un droit acquis (52), est de placer le sujet « dans une position qui lui permet d'obtenir des autres un comportement (faire faire) ou des conceptions (faire penser et faire dire) qu'ils n'auraient pas sans son intervention » (ibid.).

4 Claire Oger introduit la notion de crédibilité dans sa définition de l'autorité, en appréhendant cette dernière comme « la prétention à un « surcroît » de crédibilité, qui s'alimente certes au statut social du locuteur et/ou à sa position institutionnelle, mais qui comporte aussi une dimension discursive d'une part, contextuelle d'autre part, toutes deux étroitement liées" (2013: 237-274, cité par Monte et Oger 2015: 6). Charaudeau situe cette notion de crédibilité au niveau du sujet et de ses capacités à dire ou à faire (2005a: 52). Dans le cas d'Emmanuel Macron, la crédibilité relèverait de l'aptitude ou non à tenir un discours qui rassemble autour de ses positions, et à considérer les minorités et les différences entre les citoyens tout en maintenant un cadre républicain. Les commentateurs contestent ou confortent l'autorité du président en lui déniant ou en lui accordant la capacité de poser une conception renouvelée des rapports à l'Église qui les amènent (ou non) à penser comme lui. A travers les procédés discursifs de la (dé)construction de l'autorité du Président, ils ne donnent pas seulement à voir leur rapport à celle-ci, ils construisent aussi leur autorité propre leur capacité à porter une parole critique et à avancer des positions qui éclairent les lecteurs en ligne et reçoivent leur assentiment.

5 La méthodologie de ce travail repose sur une articulation entre les procédés discursifs de la (dé)construction de l'autorité et le contexte spécifique de la section des commentaires sur YouTube.

\subsection{L'autorité en ligne}

6 Avec l'avènement du numérique et le développement des fonctionnalités digitales sur les sites internet et réseaux sociaux, la notion d'autorité est traversée par de nouveaux questionnements. Ils dérivent de la participation des internautes au débat public et de leur rapport à des formes d'autorité préexistantes ou nouvelles.

7 Dans le prolongement de la culture médiatique contemporaine, Marcel Burger (2018: 10) souligne que la question de la légitimation des internautes ordinaires à médiatiser leur propre expertise mérite une attention particulière. Candel et Gkouskou-Giannakou 
ont montré le rôle des dispositifs numériques pour l'institution et la construction de cette légitimité à prendre la parole en ligne et à construire une identité propre. Selon les auteurs, l'autorité s'établit «dans la relation asymétrique entre les acteurs au sein des dispositifs» (2017: 21). Dans les débats politiques en ligne, la légitimité et la crédibilité des internautes passe par la construction d'un ethos (Amossy 2010), un ethos de citoyen, et par la revendication de formes diverses d'expertise. Appréhendés par Franck Babeau comme des outils d'expression politique, voire même des marques d'approbation idéologiques, les commentaires, les likes et les liens hypertextes nous donnent accès à la parole des citoyens "ordinaires" (Babeau 2014) au sujet de thématiques qui circulent dans l'espace public.

En développant l'analyse des pratiques collectives dans un article sur la fabrication de l'autorité, Domenget et Segault montrent que les internautes fondent leur énonciation moins sur la création de nouvelles autorités énonciatives que sur des formes d'autorité existantes (2017 : 93). Mais qu'en est-il des commentateurs sur la plateforme de partage de vidéos YouTube? Nous montrerons qu'en confrontant ou confortant l'autorité du Président de la République mais aussi l'autorité de la loi de 1905 fréquemment mobilisée dans les commentaires, ils appuient leur argumentation sur des autorités préexistantes (la triade de Max Weber). Ainsi, ils légitiment leur prise de parole et justifient leur positionnement au sein de la polémique. Les internautes construisent toutefois également de nouvelles formes d'autorité en ligne qui se manifestent sous les traits d'une identité de citoyen, voire d'opposant politique, à même de faire entendre ses opinions.

\subsection{Emmanuel Macron et la question de l'autorité}

9 Depuis son élection le 7 mai 2017 par suffrage universel direct, Macron est détenteur d'une autorité institutionnelle. Sa déclaration au sujet de la réparation du lien entre l'Église et l'État est donc marquée par une dimension performative fondée sur son identité présidentielle. L'enjeu de la polémique se situe autour du rôle de gardien de la Constitution qui lui incombe dès son entrée en fonction. En effet, le respect de la loi et des valeurs républicaines est intimement lié à la légitimité de ses prises de parole et son autorité, loin d'être suprême, doit se conformer au cadre défini par la Constitution française. Son discours à la Conférence des évêques de France, ou du moins la phrase relayée dans les médias, fait émerger la question des limites de l'autorité présidentielle en regard de la loi et de son interprétation.

10 La question est d'autant plus intéressante que depuis le début de sa campagne, Macron a revendiqué une certaine conception de l'autorité. Dans leur ouvrage intitulé Le poids des mots du Président. Ce que la parole de Macron dit de lui, les auteurs livrent une analyse lexicale de la mise en scène socio-langagière de son autorité par ses déclarations sur la verticalité inhérente à la fonction présidentielle (Gaboulaud et Lechevallier 2018 : 77). La construction d'un ethos présidentiel passe par une mise à distance qu'il assume, comme souligné lors d'un discours en juin 2017 lors duquel il évoque la "part symbolique" d'un chef d'État "qui suppose un cérémonial, une distance, une verticalité $»^{5}$. Antérieurs à la mise en ligne de la vidéo de notre corpus, ces déclarations résonnent dans l'interdiscours dans une perspective dialogique et participent à la construction de l'ethos préalable d'Emmanuel Macron. 
11 Face à cette conception forte de l'autorité, les commentaires des internautes sur l'autorité de Macron sont d'autant plus intéressants et font émerger une parole citoyenne qui peut être décrite comme autant de discours d'opposants politiques mobilisant des stratégies discursives et des argumentaires variés.

\section{L'espace des commentaires sur YouTube}

\subsection{Le cadre technique}

Le cadre participatif de la plateforme YouTube (Bou-Franch, Lorenzo-Dus et GarcesConejos Blitvich 2012) offre aux utilisateurs la possibilité de confronter leurs opinions et de construire une communauté autour de certaines affinités et valeurs (Lange 2008, Shifman 2011). Considérée comme une « scène communicationnelle où se manifeste de diverses façons le politique » (Douyère et Ricaud 2019: 26-27), la plateforme accueille des nouvelles formes d'expression du discours politique (20).

Comme l'ont montré plusieurs études (Amadori 2012, Douyère et Ricaud 2019), le commentaire politique sur YouTube présente de nombreux traits communs aux textes publiés sur les forums politiques en ligne (Marcoccia 2003), tantôt perçus comme des espaces démocratiques en puissance (Dumoulin 2002), tantôt critiqués pour leurs contenus haineux et leur tendance à l'homogénéité idéologique (Lev-On et Manin 2006 : 204). Ce phénomène d'homophilie est moins visible dans les réactions aux vidéos publiées par une chaîne médiatique telle que Le Monde et l'on discerne des positions plus diversifiées. En effet, la répartition des réactions au contenu de la vidéo de notre corpus indique 656 "j'aime» et 315 «je n'aime pas». Bien que ces indications quantitatives mettent en lumière un nombre de réactions positives deux fois plus élevé, il n'est pas aisé de savoir si les personnes qui ont pressé sur le bouton like l'ont fait dans le but de marquer leur appréciation du type de contenu délivré par la chaîne YouTube Le Monde ou s'il s'agit d'une réaction à la déclaration d'Emmanuel Macron intégrée au titre de la vidéo.

Les commentaires politiques, souvent brefs, n'excèdent que rarement une dizaine de lignes et sont appréhendés par Laura Calabrese Steimberg comme un objet particulièrement complexe et un espace discursif hybride, tant au niveau du registre, des marqueurs d'interlocution présents que de la portée pragmatique des énoncés (2016: 142). Considéré de manière doublement dialogique, le commentaire en ligne répond à un discours antérieur et invite à une potentielle réponse (ibid. : 143).

En raison de sa grande popularité, la plateforme YouTube est marquée par une coexistence de positionnements divers. La tendance au vase clos identifiable sur des forums en ligne est également visible dans les réactions à des vidéos publiées par des partis politiques ou des groupes idéologiques. A cet égard, une vidéo mise en ligne par la chaîne officielle de la France Insoumise est caractérisée par des réactions inégales : 449 réactions positives pour huit réactions négatives ${ }^{6}$. Toutefois, les vidéos publiées par les chaînes d'information du service public telle que la chaîne du quotidien Le Monde, qui relaie la vidéo sur laquelle nous travaillons, sont marquées par la coexistence de discours divergents.

Saisir la hiérarchisation énonciative sur YouTube est particulièrement complexe et nous nous référons aux travaux de Marta Dynel, qui en recensent trois niveaux. Le premier concerne l'interaction représentée au sein de la vidéo, le deuxième 
l'interaction réalisée par le partage de la vidéo, et le troisième correspond à l'interaction établie à l'échelle des commentaires (Dynel 2014). Le présent article se concentre principalement sur le troisième niveau de l'interaction constitué des commentaires monologaux rédigés en réponse à la vidéo et des fils de conversation qui leur sont enchâssés, les commentaires dialogaux ou polylogaux (Amadori 2012).

\section{Plateforme YouTube}

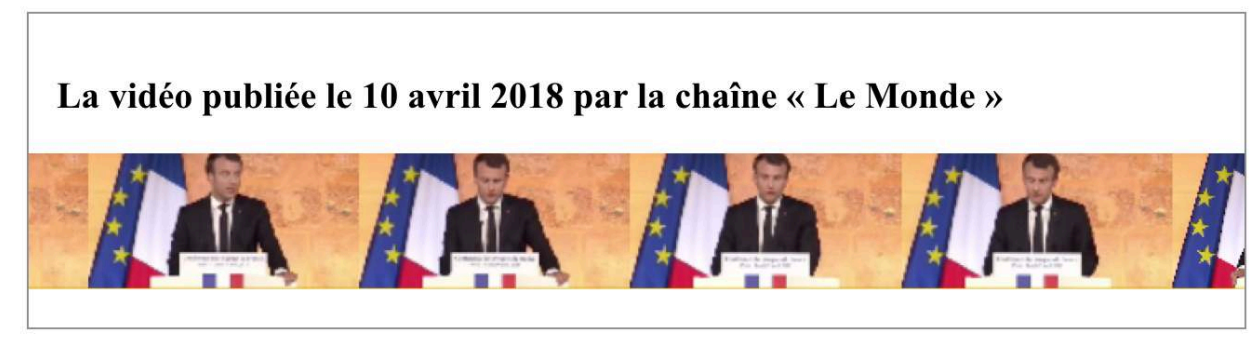

\section{0 commentaires au total}

170 commentaires monologaux

370 commentaires dialogaux ou polylogaux

\section{Hiérarchisation énonciative sur YouTube}

Souvent considéré de manière critique en raison de la violence verbale qu'il contient, le commentaire en ligne est marqué par une stéréotypisation négative. Dans Analyse du discours numérique, Marie-Anne Paveau procède à une étude des nombreuses facettes de ce "technodiscours second produit dans un espace dédié scripturalement et énonciativement contraint au sein d'un écosystème numérique connecté » (Paveau $2017: 40$ ). La chercheuse propose une typologie du commentaire en ligne et distingue : le commentaire relationnel (de type phatique), conversationnel (qui propose un véritable contenu) et moins généralement pris en compte dans la recherche sur les discours numériques : le commentaire délocalisé (sur d'autres espaces numériques) et finalement le commentaire-partage (appelé aussi « pseudo-commentaire) (ibid. 45-49).

Structurellement, le commentaire numérique est "lié au discours premier qu'il prédique » (55). Ainsi, chaque intervention peut être interprétée comme une réaction à l'impulsion donnée par la vidéo mise en ligne. Ce trait constitutif proprement natif du web répond à une dynamique d'« augmentation énonciative et discursive " (43). Les internautes sont invités à « Ajouter un commentaire public » et à participer ainsi à la "discussion » sur la thématique abordée. Ce point est significatif dans le rapport qu'ils entretiennent avec l'autorité en ligne puisqu'ils sont légitimés par le dispositif à prendre la parole.

Contrairement à d'autres espaces numériques dont les paramètres restreignent la visibilité des réactions, (les comptes ou sites web privés ou les modalités d'abonnements de certains journaux en ligne par exemple), YouTube permet à ses utilisateurs de publier du contenu public, visible ${ }^{7}$ et non payant. Ces traits sont 
significatifs dans le rapport avec la communauté des utilisateurs. L'activation ou la désactivation de la fonctionnalité des commentaires par les chaînes YouTube donne également des indications quant à la relation aux thématiques traitées dans les vidéos. Si certaines chaînes désactivent les réponses et désamorcent de potentielles critiques, d'autres encouragent au contraire à nourrir le débat.

\subsection{Les commentateurs et l'autorité}

Pour Douyère et Ricaud, la plateforme YouTube est « un espace de légitimation et de reconnaissance de pratiques et de voix, auprès de (supposés) jeunes publics, ou par de nouveaux acteurs, renouvelant les formes de communication dans l'espace public " (2019: 18). Comme le révèle la mission de la société YouTube ${ }^{8}$, ces « voix » sont invitées à s'exprimer et à être entendues. Grâce aux fonctionnalités de la plateforme et à ce que Patrick Charaudeau a identifié comme "un mécanisme de reconnaissance d'un sujet par d'autres sujets, au nom d'une valeur qui est acceptée par tous » (2005a : 50), la voix des internautes acquiert une légitimation. Nous soulignons ici l'importance de la dimension collective qui ratifie le droit à s'exprimer, élevé au rang de valeur intrinsèque sur YouTube.

21 participe à l'accueil de positionnements. Ainsi, légitimés par la plateforme à partager leurs opinions, la construction de leur autorité semble se situer autour d'un travail de crédibilisation de leur propre parole.

Afin de saisir le rapport à l'autorité du Président de la République dans notre corpus en ligne, nous faisons appel à la notion de rôle envisagé comme " un noyau de prévisibilité sociale, un ensemble typique de manières d'agir et de parler dans des situations typiques" (Eraly 2015: 30). S'inscrivant toujours dans une dimension institutionnelle, il « fait l'objet d'attentes de rôle de la part des partenaires de la relation " (ibid.). Cette perspective interactionnelle nourrit notre analyse des spécificités numériques d'un corpus natif sur YouTube.

Les internautes se positionnent en regard notamment du rôle du Président. En effet, pour une partie d'entre eux, ce qui est attendu d'un Président est intimement lié au principe de neutralité religieuse de l'État. Des commentaires métadiscursifs signalent ce qu'ils considèrent comme une forme déviante d'un rôle énonciatif (exemple A) ou d'un discours (exemple B et $C$ ) attendus :

\footnotetext{
A : C pas president quil aurait du faire, mais pape (Alexandre Le Roux)

$\mathrm{B}:$ Ce n'est pas un discours c'est un prêche... (PLEASE PLEASE)

C : Il nous fait la messe maintenant? (christian26046)
}

En ayant recours à la forme de l'opposition qui souligne un écart par rapport à une norme, les auteurs critiquent le type d'énonciateur et l'appartenance générique du discours public du Président afin de décrédibiliser ce dernier et de se positionner en tant qu'analyste légitimé par le cadre communicationnel et la communauté en ligne. Le choix de catégories religieuses appliquées à Emmanuel Macron et à son discours assimilent ironiquement le Président à un prêtre (voire un chef suprême de l'Église catholique) et soulignent son appartenance au domaine du religieux, et non à celui de l'État. Ici, la dépossession du rôle présidentiel au profit d'une nouvelle identité de chef religieux, a pour fonction de délégitimer le discours de Macron. 

fondamentale dans le débat politique, est conditionnée par la sincérité ou la transparence, la performance et l'efficacité du locuteur (2005a : 91). Dans une démarche inverse, l'entreprise de décrédibilisation menée par les internautes est caractérisée par la dénonciation de l'incapacité à remplir ces conditions. Comme le souligne Charaudeau, «se révéler menteur, incapable d'honorer ses promesses ou d'obtenir les objectifs poursuivis ne peut que discréditer le sujet "(ibid.). Ainsi, les procédés discursifs mis en œuvre par les commentateurs visent à mettre en lumière l'hypocrisie du Président, son inconsistance ou encore son incompétence. Notons à ce propos que la dénonciation des hypocrisies politiques apparait comme un trait caractéristique de l'engagement politique des publics sur les réseaux sociaux (Burger, Thornborrow et Fitzgerald 2017).

L'intervention de boulanger44800 juxtapose la fonction présidentielle de "gardien de la constitution ", présupposant l'autorité de la Constitution de la République française du 4 octobre 1958 et le rôle attendu d'un Président de respecter et protéger son contenu :

Macron, gardien de la constitution devrait la relire : « la République ne RECONNAIT, ne salarie, ne subventionne aucun culte !!! » (boulanger44800)

L'interprétation métadiscursive livrée par l'internaute Frezendv indique en plus une instrumentalisation électorale: "Discours qui a pour but d'avoir des votes supplémentaires et d'apaiser les tensions entre l'état et l'église, honteux » (Frezendv). «honteux » fonctionne comme la conclusion de l'argumentation, invalidant ainsi la condition de sincérité nécessaire à la crédibilité du Président. Qui plus est, le commentaire l'accuse de trahir sa mission en mettant son intérêt électoral propre audessus de la protection des grands principes de la République.

pose que le commentaire métadiscursif est « un lieu de renégociation des pratiques un double mouvement qui vise à rejeter l'autorité de la parole adverse et à démontrer leur capacité à faire prévaloir leur propre point de vue.

Président est caractérisé par sa conformité à une norme sociale établie. Dans cette interprétation, maintenir un lien avec les différents cultes fait partie du «cahier des charges » présidentiel et relève du domaine de l'attendu. L'étude de notre corpus met en lumière l'élaboration d'un discours de dédramatisation et d'un appel à la tradition afin de légitimer l'autorité du chef de l'État et de se construire une position de commentateur critique.

\section{La décrédibilisation du Président de la République}

internaute mobilise les textes de loi ${ }^{9}$ dans une visée argumentative (Amossy 2014, 2018) afin de décrédibiliser l'autorité présidentielle. L'inscription du rôle attendu de Macron dans le registre du devoir moral (le verbe « devoir » au conditionnel) permet de mettre en lumière une faille et participe ainsi à discréditer les ethè de sérieux, de vertu et de compétence (Charaudeau 2005a: 92-96) indispensables à un dirigeant. Ici, le

Argumentation et Analyse du Discours, 26 | 2021 
processus de décrédibilisation est étroitement lié à une démarche de délégitimation du Président de la République puisqu'il présuppose la non-acceptation d'un discours remettant en question la Constitution. Le rôle de gardien attendu apparait alors comme le prérequis du droit de gouvernance.

\subsection{L'argument par l'absurde}

L'une des stratégies mobilisées par les commentateurs afin de s'opposer à la parole du Président est le recours à l'argument par l'absurde, procédé aux fonctions proches de l'ironie (Charaudeau 2006). Ainsi, l'écart entre le discours du 9 avril 2018 et ce qui est attendu d'un chef de l'État est présenté comme inacceptable.

Un internaute a recours à une succession de questions rhétoriques pour montrer sa stupéfaction :

Comment ça le lien est abîmé il y en avait un? Et la Séparation de l'Eglise et de l'Etat alors? Il veut l'abolir? Nous faire devenir comme les Etats-Unis avec leur Republicains qui disent que c'est Dieu qui leur ordonne de faire la guerre ? J'en crois pas mes yeux. (Hodoss)

Si l'on suit la logique argumentative de l'auteur, un lien inexistant ne peut pas être abîmé. De fait, la déclaration de Macron n'a pas lieu d'être et le caractère absurde de celle-ci est mis en exergue. Par la mention de la loi de 1905 de Séparation de l'Église et de l'État et du rôle actanciel du Président dans une éventuelle abolition du texte juridique («Il veut l'abolir?») l'internaute construit l'image d'un dirigeant désireux de dominer par la force et invalide ainsi la condition de sincérité essentielle pour être jugé crédible.

En ayant recours au procédé discursif de la citation, Hodoss fait appel à une autorité plus haute dans une visée argumentative. Définie par Maingueneau, la citation d'autorité permet à un locuteur de s'effacer « devant un Locuteur superlatif qui garantit la validité de l'énonciation» (Maingueneau 1991: 138). En mobilisant une instance énonciative reconnue, ces énoncés citationnels donnent l'impression d'être « la Parole même saisie en sa source » (138). Il est important de souligner le caractère normatif d'un texte juridique dont la fonction est de définir les droits et les devoirs des citoyens. Dans le cas de la délégitimation de la parole présidentielle, la mobilisation de la citation de la loi de 1905 apparaît comme une citation d'appui, qui a selon Cazalbou pour fonction d'inscrire la pièce rapportée «dans une filiation ou au contraire de s'en démarquer» (2012:13). Par la dénonciation d'une infraction à la loi, l'internaute fait émerger l'absurdité de la déclaration du chef d'État et déconstruit à nouveau son image de gardien de la loi.

De plus, l'évocation du rapport au religieux dans le contexte américain fonctionne ici comme un argument par l'exemple qui présuppose «l'existence de certaines régularités dont les exemples fourniraient une concrétisation » (Perelman 1977 :119), permettant ainsi au locuteur d'opérer une généralisation dans une visée de persuasion. De manière implicite, les caractéristiques négatives de l'exemple sont appliquées au cas de la France afin de dénoncer l'absurdité de la démarche de Macron et de provoquer un sentiment d'effroi, renchéri par l'expression idiomatique « J'en crois pas mes yeux » qui marque le caractère stupéfiant de l'observation. Ce commentaire illustre par le recours à l'exemplum les conséquences jugées néfastes par l'internaute. Là encore, l'auteur 
invalide la condition d'efficacité nécessaire au Président pour revendiquer un ethos de crédibilité.

D'autres internautes ont également recours à un exemple d'anticipation dans le but d'esquisser le caractère absurde de l'action d'Emmanuel Macron et ainsi de le décrédibiliser :

Bah pour réparer ce lien entre l'Eglise et l'Etat y'a qu'à remettre le catholicisme religion d'état et puis c'est tout (Dark Shinigami)

La dimension de l'absurde est amplifiée par une conclusion présentée comme un état de fait imminent :

Bientôt la charia ds la Constitution. (FROMAGE DOUX)

De manière implicite, le discours de Macron apparaît ici comme une première étape aboutissant à l'entrée de la charia dans la Constitution française.

En esquissant de manière fictive le monde d'après de manière négative, les internautes mobilisent le pathos par la convocation implicite d'images négatives liées au cléricalisme dans le premier exemple et à la charia dans le second. La mobilisation de la doxa a alors pour fonction de provoquer un sentiment de peur par anticipation et permet aux internautes de présenter la démarche de réparation du lien comme illogique et inacceptable.

\subsection{Les attaques personnelles}

L'argumentation ad personam tient une place importante dans les commentaires sur YouTube (Amadori 2012). Afin de tenter de dégager les mécanismes de la décrédibilisation de la parole présidentielle, nous nous concentrerons sur deux types d'attaques personnelles récurrentes dans notre corpus : les insultes et la dérision.

\subsubsection{La forme discursive de l'insulte}

41 La forme discursive de l'insulte poursuit des fonctions argumentatives visant à déconstruire l'identité du destinataire. La déshumanisation est une stratégie argumentative fréquemment utilisée par les internautes en ligne. Celle-ci peut prendre la forme d'une réification comme dans le commentaire de Jean Naimar qui réifie le Président en le présentant comme une marionnette :

Peu importe de quoi il parle, ce freluquet marionnette a vraiment l'air d'une pédale fragile. Aucune crédibilité (Jean Naimar)

42 La métaphore de la marionnette lui permet de construire une image marquée par la fragilité qui met à distance le destinataire de la force et de la virilité communément attribuées à un dirigeant politique. L'insulte marque l'incapacité du Président à remplir la condition de performance (Charaudeau 2005 : 91) nécessaire à sa crédibilité. Nous pouvons nous demander si cela ne participe pas à une démarche plus large de délégitimation qui aurait pour objectif de remettre en question le droit d'exercer le pouvoir. C'est précisément le constat exprimé de manière explicite par la phrase nominale en fin de commentaire : « Aucune crédibilité ».

Le recours à la bestialisation permet également aux internautes de déshumaniser la figure présidentielle afin de la discréditer. Pour Fodie Kebe, Emmanuel Macron est un « chacal avide de pouvoir»:

Il essaye de reconquérir le vote des chrétiens ce chacal avide de pouvoir 
En dressant le portrait du Président sous les traits non seulement d'un animal mais d'une bête sauvage, la disqualification morale « franchit un palier» (Ravat 2019 : 95). Le pouvoir prend ici la forme d'un animal prédateur qui chercherait à tromper les « chrétiens». Par ce procédé argumentatif, l'auteur du commentaire condamne les motivations morales d'Emmanuel Macron et porte atteinte à la condition de sincérité et de transparence nécessaire pour se montrer digne de crédit.

Le rapport entre la figure discursive de l'insulte et la condamnation d'une instrumentalisation politique est aussi observable dans l'exemple suivant :

Macron et la manipulation ça fait 2. Il veut récupérer l'église pour gagner en popularité et mieux la contrôler, ni plus ni moins. C'est un psychopathe narcissique ce type (azerty azertu)

La première attaque vise à dénoncer la démagogie d'Emmanuel Macron par la manipulation et la récupération afin de "gagner en popularité ». Si la démarche de persuasion est inhérente au discours politique, l'image d'un dirigeant doit être marquée par des valeurs de vertu qui répondent à ce que Charaudeau définit comme l'attente fantasmatique de la part des citoyens d'être représentés par un dirigeant «qui soit un modèle de droiture et d'honorabilité » (2005: 95). De fait, il ne peut se permettre d'être associé à une image liée à la manipulation et aux tentatives de contrôle. En dépossédant le Président de la République des traits de vertu attendus par l'instance citoyenne, l'auteur du commentaire porte atteinte à son ethos de crédibilité.

47 La seconde attaque relève de l'insulte et a pour fonction d'invalider la condition d'efficacité comprise comme «la preuve que le sujet a les moyens d'appliquer ce qu'il promet et que les résultats sont positifs » (ibid.: 92). En attribuant au Président les caractéristiques de la maladie psychiatrique : «psychopathe narcissique », l'internaute déconstruit l'identité institutionnelle de Macron en la remplaçant par une identité déviante marquée par la pathologie. L'ad personam vient ici conclure l'argumentation visant à la décrédibilisation.

\subsubsection{La forme discursive de la dérision}

8 La décrédibilisation de l'autorité présidentielle passe également par un jeu de contraste pointant le contenu du discours d'Emmanuel Macron et l'incompatibilité de la « réparation du lien entre l'Église et l'État » en regard de l'histoire :

Quoi ?? Mais on est un état laïque! L'état et l'Eglise sons séparés depuis des siècles... (Daphnée Dereux-Suin)

Dereux-Suin marque sa stupéfaction par un discours exclamatif et fait appel à deux sources d'autorité : la nature laïque de l'État définie par la Constitution de 1958 et la loi de Séparation de l'Eglise et de l'Etat de 1905 évoquée par l'auteure de manière implicite. Elle ancre son propos dans l'histoire et la nature même de la République et montre ainsi que l'écart par rapport à la tradition est absurde. Elle mobilise l'autorité traditionnelle définie par Weber comme reposant sur « la croyance quotidienne en la sainteté de traditions valables de tout temps et en la légitimité de ceux qui sont appelés à exercer l'autorité par ces moyens" (1992). Ainsi, l'auteure convoque de manière implicite des valeurs communes dotées d'une "force de vérité " (Charaudeau 2005a : 162) ou, pour reprendre les termes de Monte et Oger, de la forme de l'évidence qu'ils envisagent comme un moyen d'étayer l'autorité (2015: 11). La rédactrice du commentaire inscrit son discours dans l'imaginaire de la «tradition» (2005a : 163). La 
laïcité de l'État et la Séparation entre l'Église et l'État sont érigées en valeurs à défendre. Par ce tour de passe-passe maniant les modalités de l'implicite, Macron est désigné comme une menace, ce qui invalide sa crédibilité présidentielle. En dénonçant une infraction à la tradition républicaine et à l'histoire de France, les internautes mettent en lumière l'absurdité de la déclaration d'Emmanuel Macron par un jeu de contraste et déconstruisent son ethos de dirigeant fidèle à ses origines, ses textes de loi et l'ensemble des citoyens.

Le recours à la forme de la dérision est ainsi pour de nombreux internautes un moyen efficace de porter atteinte à la crédibilité du Président en mettant en lumière une facette négative de sa personne ou de son discours. La forme discursive de la dérision est aussi visible dans la reprise d'expressions énoncées par Macron. Dans la circulation des discours, les représentations de sa parole publique mettent en évidence des expressions désuètes ${ }^{10}$, «la poudre de perlimpinpin » ayant reçu le plus de visibilité dans les médias et sur les réseaux sociaux après le débat de l'entre-deux-tours face à Marine Le Pen. Un internaute reprend cette expression :

Tout ça c'est de la poudre de perlimpinpin (tntg5)

51 En réaction à ce commentaire, Titi Ave Maria emprunte selon le même procédé dialogique l'expression vieillie "carabistouille », prononcée également par Emmanuel Macron. Par un procédé d'hyperbole introduit par "je dirais même", l'internaute montre qu'il suit la même logique discursive. Ces auteurs font appel à l'humour et à l'implicite pour critiquer de manière métalinguistique le discours de Macron. Toutefois, ces deux interventions se concentrent sur deux aspects distincts du discours. En ayant recours à « la poudre de perlimpimpin », tntg5 rapproche les paroles du Président d'un tour de passe-passe et dénonce ainsi non seulement sa dimension superficielle mais aussi l'entreprise de tromperie du public. Le second commentaire quant à lui critique le contenu des propos en les qualifiant de balivernes.

52 Ces procédés discursifs permettent aux internautes de déconstruire l'image d'un Président proche du peuple qui se fait bien comprendre ; ils critiquent le discours vieilli et poussiéreux du dirigeant. En empruntant ce figement discursif, « tntg5 » souligne la distance entre Macron et son électorat et porte ainsi atteinte à son autorité sur celui-ci.

Il est intéressant de constater que le commentaire de tntg5 est le plus «liké » de l'ensemble des 540 réactions qui font suite à la vidéo mise en ligne par la chaîne Le Monde. Au total, 181 internautes ont aimé ce commentaire, contribuant ainsi à le placer en première place du tri proposé par l'algorithme de la plateforme (selon le tri par pertinence sélectionné par défaut) et à lui donner de ce fait une forte visibilité. L'écart entre ces expressions figées relevant du passé et les discours natifs sur YouTube semble être exacerbé par la dimension générique du discours en ligne, représenté dans l'imaginaire collectif comme un lieu de discours moderne.

Nous avons pris le parti d'aborder l'opposition à l'autorité présidentielle par le biais de la décrédibilisation. Toutefois, nous pouvons nous demander si certains de nos exemples ne dévoilent pas également une démarche de délégitimation. La déconstruction de l'ethos de crédibilité a pour fonction de dénoncer l'écart entre la figure d'Emmanuel Macron et celle qui est attendue d'un chef d'État, invalidant alors son droit à exercer le pouvoir. Ainsi, par différents procédés de décrédibilisation, les internautes opposés au Président de la République renforcent leurs arguments et participent ensemble à une entreprise de délégitimation. 


\section{La légitimation de l'autorité du Président de la République face aux détracteurs} procèdent à la légitimation de son autorité fondée sur la dédramatisation du discours tenu lors de la Conférence des évêques de France. Le recours à la citation de la loi de Séparation de l'Église et de l'État et l'interpellation de la communauté YouTube s'appuient sur une légitimité juridique dans le premier cas et collective dans le second.

\subsection{La reconfiguration du discours controversé}

Si les commentaires critiquant le discours de Macron répondent principalement au contenu de la vidéo, il est intéressant de constater que ceux qui le soutiennent sont marqués par la prise en compte des arguments des détracteurs. Des procédés argumentatifs divers permettent aux internautes de procéder à une reconfiguration de la controverse en accordant une place au discours adverse par la polyphonie.

L'exemple qui suit offre un exemple intéressant d'un appel collectif visant à « arrêter la paranoïa » :

Arrêtez d être scandalisé tout président ferait la même chose pour rassurer l église et les pratiquants et des speaches comme ça sans aucun intérêt tous les présidents au monde en font chaque semaine, et $c$ est pas avec un truc qui $n$ intéresse personne qu il essayerait de cacher quelque chose, arrêtez votre paranoïa (O O)

L'auteur inscrit le discours présidentiel dans le registre de la normalité: «tout président ferait la même chose ", des speeches comme ça sans aucun intérêt " tous les présidents au monde en font chaque semaine ». Par la mobilisation de la règle de justice de Perelman (1998) qui vise à mettre en lumière le parallèle « si A est vrai pour B alors A est aussi vrai pour C ", l'internaute affirme la conformité du Président aux attentes suscitées par la fonction de chef d'État à travers le monde. Par un processus argumentatif de généralisation, le discours controversé est ainsi dédramatisé et l'image de Macron est protégée.

Sous une forme dialogique de questions-réponses, Sarah Dumas désembraie les arguments des opposants et reconfigure le discours de la controverse comme un moment de respect «touchant»:

Je trouve scandaleux la polémique faite autour ce discours. Où est le mal de faire un discours devant les évêques? On a vu un prêtre égorgé devant ses paroissiens dans notre pays et 1 église est restée digne. $C$ est la religion la plus attaquée en France (même si les musulmans sont aussi stigmatisés) mais il est de bon ton de se moquer des ktos. Alors moi ce moment de respect je le trouve touchant. Ca equilibre par rapport à d autres communautés. Et puis un president peut avoir la foi. Pourquoi ce serait de la recuperation? Et je ne vois pas en quoi ce discours interfere avec la laïcité . Je conseille à ceux qui critiquent de lire la loi de 1905

En attribuant au discours d'Emmanuel Macron la valeur du respect, l'auteure participe à la (re)construction de l'image du chef d'État. A l'aide de plusieurs verbes modaux à la première personne, Sarah Dumas construit son positionnement et montre en quoi la polémique qui a émergé relève du scandale - «d'une conduite qui provoque la réprobation, l'indignation, le blâme » (TLFi), dénonçant ainsi les arguments adverses. Dans cette démarche de reconfiguration, la mention de la loi de 1905 vient appuyer le positionnement de l'auteure. La question rhétorique « Où est le mal de faire un discours

Argumentation et Analyse du Discours, 26 | 2021 
devant les évêques?» fait émerger l'absence d'interdiction morale de s'adresser au public cité et par extension le «droit de faire » ce discours. En ce sens, cette stratégie argumentative participe à la (re)légitimation d'Emmanuel Macron.

En réponse aux arguments adverses qui dénoncent l'infraction à la tradition, l'exemple de Dale Cooper illustre une mobilisation de l'histoire de la France dans une visée argumentative :

Je ne suis pas croyant mais ce qu'il dit est très juste : l'histoire de France a été écrite en majorité par des chrétiens, guidés par leurs foi. Le nier serait absurde, je ne vois pas où est le problème

L'auteur met en lumière le caractère absurde d'une interprétation qui aurait pour but de nier l'histoire de France et l'autorité traditionnelle qui lui est rattachée - voir la citation de Weber (1995: 289) donnée plus haut. Les auteurs des commentaires mobilisent le lien étroit entre tradition et autorité dans leur entreprise de légitimation d'Emmanuel Macron. L'internaute Dale Cooper s'appuie ici sur la dimension patrimoniale et historique de l'Église. Selon Yann Raison du Cleuziou, le recours aux racines chrétiennes de la France a pour fonction de tenter de perpétuer un privilège catholique dans l'accès à l'État et dans la régulation du champ religieux national (2020 : 75). Rejetant le caractère exceptionnel du discours de la Conférence des évêques, les internautes le reconfigurent en soulignant sa dimension attendue, généralisée dans le monde et qui s'inscrit dans une continuité historique.

\subsection{L'interpellation de la communauté YouTube}

63 Si les internautes construisent leur propre autorité de citoyens à même de débattre, il est intéressant de constater qu'ils participent également à la légitimation de la parole politique des autres membres de la communauté. L'exemple suivant suit cette logique d'interpellation:

Je crois ce que je viens dire est vrai, sinon donner votre opinion (Cornetmur 04)

La dimension dialogale encouragée sur la plateforme permet aux internautes de mettre en valeur leur propos et participe à la construction d'une autorité leur permettant de s'attaquer ou au contraire de légitimer l'autorité d'un homme politique.

En partant des propos relayés dans la vidéo ou de la polémique présentée, les internautes poursuivent le débat entre eux, et montrent ainsi qu'ils sont des citoyens à même de faire entendre leurs opinions et d'argumenter. Le commentaire de Peej Myr prend la forme d'un discours mobilisateur, d'un appel à l'action et à l'opposition. En s'adressant aux Français, l'auteur élargit la portée de son discours : il ne s'adresse plus seulement à la communauté des utilisateurs de la plateforme YouTube spectatrice des échanges mais à l'ensemble des citoyens français. Sous la forme d'un contre-discours, il appelle « le peuple » à se réveiller, à combattre «les problèmes un par un par ordre d'importance", ou encore à reprendre la main. Peej Myr emprunte un lexique militaire: "quelle maîtrise de la guerre ce Macron", "bombe à fragmentation", "diversions", "attaques surprises" - et se construit ainsi une identité d'opposant politique face à Macron, présenté comme l'«ennemi». Dans une logique argumentative, il s'adresse à ses concitoyens au travers d'une succession d'appels à l'action sur le mode de l'impératif:

Reprenez la main! Faites votre actualité, c'est vous le peuple, c'est vous les concernés, c'est vous la France (Peej Myr) 
forme discursive de la répétition permet à l'auteur d'effectuer un double travail de construction identitaire. Premièrement, il renforce son ethos d'opposant politique en donnant des consignes mobilisatrices sur un ton alarmiste. Il se distingue de son auditoire en se présentant de manière implicite comme un dirigeant potentiel. De plus, sa construction identitaire passe par l'affirmation de l'auditoire: «le peuple», «les concernés », «la France ». L'emploi de la figure métonymique «la France » permet à Peej Myr de construire une image positive de son auditoire et de transcender les limites de la communauté en ligne.

Potentiellement, les commentaires des internautes peuvent acquérir une visibilité particulièrement importante et participer à l'émergence d'une parole publique en ligne. Sur YouTube, les commentaires sont rattachés à des contenus vidéos et ne sont pas identifiés dans le flux d'un profil d'utilisateur comme sur une messagerie telle que Whatsapp ou Facebook Messenger. Ce trait distinctif des réseaux sociaux, qui place le contenu prédiqué au centre, a une influence sur la visibilité et sur le rapport des internautes à l'autorité en ligne. Toutefois, parmi l'abondance de réactions publiées continuellement, un commentaire peut obtenir, selon des conditions liées aux calculs algorithmiques et par le biais de l'investissement en ligne de l'internaute, une visibilité importante au sein de la communauté et conférer aux auteurs une autorité particulière dans le débat. Celle-ci peut être alors ratifiée par le bouton "j'aime» et par les commentaires dialogaux qui lui répondent. Propulsés par la logique algorithmique et la participation collective, les internautes peuvent espérer gagner en visibilité et ainsi mettre en valeur leur identité en ligne.

\section{Conclusion}

68

La réforme de la loi de Séparation de l'Église et de l'État est l'une des questions fréquemment débattues dans l'espace public depuis le début de la campagne présidentielle d'Emmanuel Macron. Celui-ci est souvent resté flou sur l'agenda et les modalités de l'amendement de la loi. Après avoir fait marche arrière au terme du grand débat national en pleine crise du mouvement des Gilets Jaunes, le Président de la République annonce toutefois le 30 septembre 2020 un nouveau projet de loi sur les séparatismes ${ }^{11}$ qui vise à mettre à jour la loi de 1905. Ce tâtonnement caractéristique du positionnement d'Emmanuel Macron sur la laïcité ${ }^{12}$ est révélateur du dilemme inhérent à sa fonction : d'un côté celle de gardien de valeurs traditionnelles et de l'autre, celle de réformateur d'une société en mouvement. En réponse à la polémique suscitée par sa volonté de réparer le lien entre l'Église et l'État lors de la Conférence des évêques de France, des internautes mobilisent les fonctionnalités des commentaires de la plateforme YouTube afin de déconstruire ou reconstruire l'autorité présidentielle et de se conférer une autorité de commentateurs politiques.

Nous avons montré que les internautes opposés au Président ont recours à des procédés argumentatifs variés tels que la reductio ad absurdum ou l'ad personam afin de déconstruire l'ethos de crédibilité nécessaire à l'exercice de la fonction présidentielle, et de démontrer qu'il n'est pas investi d'une autorité qui lui permet de proposer un renouvellement des rapports entre l'Église et l'État. Si nous avons pris le parti de nous concentrer sur la démarche de décrédibilisation menée par les rédacteurs, notre analyse a cependant révélé que certains d'entre eux vont jusqu'à la mise en cause de la légitimité d'Emmanuel Macron dans ses fonctions institutionnelles au sens large.

Argumentation et Analyse du Discours, 26 | 2021 
70 De leur côté, les internautes favorables à la déclaration d'Emmanuel Macron ont recours à un procédé de dédramatisation du discours controversé et à la mobilisation de la loi de 1905 afin de tenter de rétablir l'autorité présidentielle mise à mal. La prise en compte dialogique des autres utilisateurs de la plateforme renforce non seulement l'autorité de commentateurs politiques des scripteurs mais aussi celle de l'ensemble de la communauté en ligne.

71 Ces discours sur YouTube révèlent, entre autres, les potentialités des RSN liées aux formes d'appel à l'action adressées à une communauté d'internautes censée partager les mêmes opinions face à l'autorité présidentielle. Ils peuvent ainsi asseoir la légitimité de la parole citoyenne sur une dimension collective non spécifiée. En effet, les commentaires en ligne sont caractérisés par de nouveaux mécanismes de médiatisation en ligne et d'actions collectives (Babeau 2014: 146). Dans ces espaces numériques publics et visibles (Paveau 2017), le lien entre le statut institutionnel et l'autorité se voit reconfiguré, la légitimité et la crédibilité du Président faisant l'objet de nombreuses mises en discussion. Ces échanges polémiques mettent en scène des conflits d'autorité qui permettent aux internautes non seulement de déconstruire l'autorité présidentielle mais aussi de construire leur propre crédibilité et légitimité, potentiellement renforcées par les paramètres technodiscursifs mis à disposition par la plateforme tels que le bouton like, le partage de contenu ou encore l'augmentation de la visibilité par les algorithmes.

\section{BIBLIOGRAPHY}

Amadori, Sara. 2012. «Le débat d'idées en ligne : formes de la violence polémique sur Youtube. Signes, Discours et Sociétés 9 [En ligne] http://revue-signes.gsu.edu.tr/article/LXz7XjdmoGn60Q8Uf2t (consulté le 20 juillet 2018)

Amossy, Ruth. 2010. La présentation de soi. Ethos et identité verbale. (Paris : PUF)

Amossy, Ruth. 2014. L'Apologie de la polémique (Paris : PUF)

Amossy, Ruth. 2018. «Introduction : la dimension argumentative du discours - enjeux théoriques et pratiques ». In Argumentation et Analyse du Discours 20 [En ligne](consulté le 25 avril 2018)

Ardid, Claude, Marika Bret \& Nadège Hubert. 2020. Qui veut tuer la laïcité ? (Paris : Eyrolles)

Babeau, Franck. 2014. « La participation politique des citoyens "ordinaires" sur l'Internet. La plateforme YouTube comme lieu d'observation », Politiques de communication 3, 2014/2, 125-150

Bou-Franch, Patricia, Nuria Lorenzo-Dus \& Pilar Garces-Conejos Blitvich. 2012. «Social Interaction in YouTube Text-Based Polylogues: A Study of Cohérence », Journal of ComputerMediated Communication 17.4, 501-521

Burger, Marcel (dir.). 2018. La communication digitale : entre affordances et discours multimodaux, Cahiers de l'ILSL 55 
Burger, Marcel, Thornborrow Joanna \& Fitzgerald, Richard (éd). 2017. Discours des réseaux sociaux : enjeux publics, politiques et médiatiques (Louvain-la-Neuve : De Boek Supérieur)

Burgess, Jean \& Joshua Green. 2009. YouTube: Online Video and Participatory Culture (Cambridge UK: Polity Press)

Calabrese Steimberg, Laura. 2007. "Quel(s) objet(s) de discours se dissimule(nt) sous la dénomination le voile ?, L'acte de nommer : Une dynamique entre langue et discours (Paris : Presses Sorbonne Nouvelle)

Candel, Étienne \& Pergia Gkouskou-Giannakou. 2017. « S'instituer par l'écriture en ligne », Communication \& langages 192. 2, 19-26

Cazalbou, Renaud. 2012. «De la citation à l'autorité : liberté et contrainte dans le discours argumentatif », Voix et marqueurs du discours : des connecteurs à l'argument d'autorité. (Lyon : ENS Editions)

Charaudeau, Patrick. 2005a. Le discours politique. Les masques du pouvoir (Paris : Vuibert)

Charaudeau, Patrick. 2005b. « Quand l'argumentation n'est que visée persuasive. L'exemple du discours politique ». Burger M. \& G. Martel (éds). Argumentation et communication dans les médias (Québec : Editions Nota Bene), 29-49

Charaudeau, Patrick. 2006. « Des catégories pour l'humour?», Questions de communication 10, $19-41$

Charaudeau, Patrick \& Dominique Maingueneau (éds). 2002. Dictionnaire d'analyse du discours (Paris : Seuil)

Domenget Jean-Claude \& Antonin Segault. 2017. « Une pratique collective de fabrication de l'autorité », Quaderni [En ligne], 93 | Printemps 2017, mis en ligne le 05 mai 2019 (consulté le 04 janvier 2020)

Douyere, David \& Pascal Ricaud. 2019. «Présentation du dossier. Youtube, un espace d'expression politique? », Politiques de communication 13.2, 15-30

Dumoulin, Michaël. 2002. «Les forums électroniques : délibératifs et démocratiques? ", Monière, D. Internet et la démocratie : les usages politiques d'Internet en France, au Canada et aux États-Unis (Montréal : Monière et Wollank), 140-157

Dynel, Marta. 2014. « Participation framework underlying YouTube interaction », In Journal of Pragmatics, $\mathrm{n}^{\circ}$ 73, https://doi.org/10.1016/j.pragma.2014.04.001, 37-52

Eraly, Alain. 2015. Autorité et légitimité. Le sens du collectif (Toulouse : Erès coll. « Sociologie clinique »)

Gaboulaud, Adrien \& Anne-Sophie Lechevallier. 2018. Le poids des mots du Président (Paris : Editions de l'Observatoire)

Gern Laetitia. 2019. «Commenter sur YouTube : un positionnement politique complexe ». Burger, Marcel (éd). Se mettre en scène en ligne. La communication digitale, vol.2., Cahiers de l'ILSL 59, 75-89

Herring, Susan C. 2011. « Discourse in Web 2.0: Familiar, reconfigured, and emergent », Tannen D. \& A.-M. Trester, Discourse 2.0. Language and the new media, (Washington DC: Georgetown U. P.), $1-26$

Herring, Susan C. 2016. " New frontiers in interactive multimodal communication ». In Georgakopoulou G. \& T. Spilioti (éds). The Routledge Handbook of Language and Digital Communication (London-New York: Routledge), 398-402 
Lange, Patricia G. 2008. « Publicly Private and Privetely Public: Social Networking on YouTube », Journal of Computer-Mediated Communication13.1, 361-380

Lev-On, Azi \& Bernard Manin. 2006. « Internet : la main invisible de la délibération », Esprit 5, mai 2006, 195-212

Maingueneau, Dominique. 1991. L'analyse du discours. Introduction aux lectures de l'archive (Paris : Hachette)

Marcoccia, Michel. 2003. « Parler politique dans un forum de discussion », Langage et société 104, 9-55

Monte, Michèle \& Claire Oger. 2015. Discours d'autorité : des discours sans éclat(s) ?, Mots. Les langages du politique $107,5-17$

Paveau, Marie-Anne. 2017. L'analyse du discours numérique. Dictionnaire des formes et des pratiques (Paris : Hermann)

Perelman, Chaïm. 1977. L'Empire rhétorique (Paris : Vrin)

Perelman, Chaïm \& Olbrechts-Tyteca, Lucie. 1998 [1958]. Traité de l'argumentation (Bruxelles : Editions de l'Université de Bruxelles)

Raison du Cleuziou, Yann. 2020. «L'Eglise, fille aînée de la République ? Les apologies de la catholaïcité depuis les attentats de janvier 2015 », Nouveaux vocabulaires de la laïcité 469 (Paris :

Classiques Garnier), 59-75

Ravat, Jérôme. 2019. Éthique et polémiques. Les désaccords moraux dans la sphère publique. (Paris : CNRS Editions)

Shifman, Limor. 2011. «An anatomy of a YouTube même », New Media \& Society 14.2, 187-203

Smith, Simon. 2017. Discussing the News. The Uneasy Alliance of Participatory Journalists and the Critical Public (Cham: Palgrave Macmillan)

Weber, Max. 1995 [1922]. Économie et société, 2 vol. (Paris : UGE Poche)

Weil, Patrick (éd). 2007. Politiques de la laïcité au XXe siècle (Paris : PUF)

\section{NOTES}

1. Site officiel du gouvernement français : www.gouvernement.fr, article intitulé «Promulgation de la loi concernant la séparation des Églises et de l'État.

2. C'est bien l'interprétation de Marine Le Pen sur RTL, relayée dans un article du journal Le Monde publié le 10 avril 2018.: « le président d la République tente d'anesthésier les catholiques pour pouvoir demain s'attaquer à la loi de $1905 »$.

3. Le Figaro, le 10 avril 2018.

4. Lien de la vidéo publiée sur la chaîne YouTube Le Monde : https://www.youtube.com/watch? $\mathrm{v}=\mathrm{rhEpj9xQTi0}$

5. Discours au lycée agricole de Limoges-les-Vaseix, le 9 juin 2017

6. Vidéo publiée le 12 juillet 2018 sous le titre « DÉBAT SUR LA LAÏCITÉ ET LE CONCORDAT À L'ASSEMBLÉE NATIONALE ».

7. Marie-Anne Paveau distingue public et visible comme suit: « public concerne le statut technique et juridique; visible concerne la configuration discursive et la relation entre les internautes et les énoncés » (2017:44). 
8. « Notre mission est que chacun puisse faire entendre sa voix et découvrir le monde ». Page de présentation du site internet YouTube.

9. La citation signalée par des guillemets n'est pas un extrait de la Constitution évoquée dans la première partie du commentaire mais la première phrase du deuxième article de la loi de Séparation des Eglises et de l'Etat de 1905.

10. Le Parisien, 4 mai 2017 : "Débat: "poudre de perlimpinpin" et "galimatias", les expressions désuètes de Macron »

11. Le Figaro, 30 septembre 2020

12. Marianne, 6 janvier 2018 : « Laïcité : les silences choisis d'Emmanuel Macron »

\section{ABSTRACTS}

La déclaration controversée du Président de la République Emmanuel Macron au sujet de la réparation du lien entre l'Église et l'État nous offre un cas d'analyse spécifique dans lequel le rapport à l'autorité est débattu. L'article montre que la question de la déconstruction de l'autorité dans l'espace des commentaires sur YouTube passe par la mobilisation de l'argument par l'absurde et par des attaques personnelles telles que l'insulte ou la dérision. Ainsi, les internautes opposés aux dires d'Emmanuel Macron décrédibilisent son autorité et vont jusqu'à mettre en cause sa légitimité à exercer le pouvoir. De leur côté, les internautes favorables à la restauration du lien reconfigurent le discours controversé en s'appuyant sur la loi de 1905 et font appel à l'ensemble de la communauté YouTube. En utilisant les fonctionnalités technodiscursives de la plateforme, les internautes participent au débat et se confèrent une autorité de commentateurs politiques.

The controversial statement of the French President Emmanuel Macron about "repairing the link between Church and State" provides an interesting case study as far as the relationship to authority is concerned. The article shows that the deconstruction of authority in the space of YouTube comments involves mobilizing the argument of reduction ad absurdum as well as personal attacks such as insults or derision. Thus, internet users opposed to Emmanuel Macron's claims discredit his authority and go so far as to question his legitimacy to exercise power. For their part, internet users in favor of restoring the link reconfigure the controversial discourse by relying on the 1905 lawon the Separation between Church and State, and appeal to the entire YouTube community. By using the platform's technodiscursive features, You Tube users participate in the debate and confer upon themselves an authority of political commentators.

\section{INDEX}

Mots-clés: autorité présidentielle, commentaires YouTube, débat politique, décrédibilisation, loi de 1905

Keywords: 1905 law of Separation between State and Church, decredibilization, political debate, YouTube comments, presidential authority 
AUTHOR

LAETITIA GERN

Université de Lausanne 